\title{
A Conceptual Framework for Problems in Elastostatics
}

\author{
Walter Noll
}

March 8, 2011

Carnegie Mellon University, Pittsburgh, PA, USA. E-:mail: wn0g@andrew.cmu.edu .

\begin{abstract}
In finite elasticity, the traditional local boundary-value problems are rarely appropriate. More often one has to consider non-local environmental loads. This paper gives a general description of schemes for formulating such non-local problems. Interesting examples are cavity problems, special cases of which are what I call basketball problems and tire problems. The actual solution to these problems I leave to future work.
\end{abstract}

\section{Mathematics Subject Classification (2010)74B20, 74M99}

Key words: Finite elasticity. Elastic loads, Environmental loads, Formulation of Problems.

\section{Introduction}

It is a cliché that "mathematical physics" is, in part, the study of "boundary-value" and "initialvalue problems", and that such problems are "well-posed" if one can prove existence, uniqueness, and stability of solutions. This is a narrow view even when the differential equations that govern the given situation are linear; it is totally inadequate when these equations are non-linear, as will be evident from the discussions of the problems in Section 5.

In Sections 2 and 3 I will give a brief description of the concepts of a continuous body and of a load system acting on such a body. In Section 4, after defining what is meant by an elastic body, I will describe the load operator of such a body. This operator associates with each placement of 
the body the load-system necessary to keep the body in this placement. After discussing zero-load problems in Section 5, I present, in Section 6, a general framework for problems in elastostatics. The new feature of this framework is that one prescribes, not boundary data, but an environmental reaction operator which specifies how the environment would act on the body for every conceivable placement. The problem then is to find the placements, if any, for which the load operator and the environmental reaction operator have the same value.

Most of this paper cannot be understood by a reader who is not familiar with what I called, in [CMI], the Stage 3 Infrastructure of mathematics. Unfortunately, many mathematicians, physicists, and engineering scientist are sill stuck in Stage 2. In a nutshell, Stage 2 is based on the concepts of variables, coordinates, parameters, and constants. Stage 3 is based on the concepts of sets, of mappings with domain and codomain, and of mathematical structures. An idea of the difference can be obtained by reading my book Finite-Dimensional Spaces, Algebra, Geometry, and Analysis, Volume I [FDSI], especially the Introduction and Chapter 0. This book was published in 1987. A corrected version is now available, free of charge, on my website. To this day, students are subject to courses with titles such as Matrix Algebra, Vector Analysis, Tensor Analysis, and Multivariate Calculus. In my opinion, such courses deserve to be put into the trash, or, at best, in a museum.

In particular, I use the notations (00.1), (03.20), (03.21) and $f^{\leftarrow}$ on p.11 in Chapter 0 of [FDSI], as well as some notations and definitions from Section 18 and 63 of [FDSI].

My own work since 1972 requires understanding of the Stage 3 infrastructure. The ideas presented in my work cannot be described by using only Stage 2 because this infrastructure is too crude and imprecise.

Also, most of this paper cannot be understood by a reader who is not familiar with the notation and terminology of the frame-free background of continuum mechanics presented in $[\mathrm{BC}],[\mathrm{FFE}]$ ,and [FC]. especially Sections 1,2,3,4,5,8, and 9 of [BC].

I presented the basic ideas underlying this article at a meeting in Rio de Janeiro in 1978 [Rio]. The present paper differs from [Rio] in that it is more precise and elaborate, and hat it uses better concepts and notations because it is is based on the recently developed background mentioned in the previous paragraph. In addition, in Section 6, it includes a general theory of elastic bodies with gas filled cavities. Special cases of it are what I call the basketball problem and the tire problem. Finally, there were some serious errors in [Rio] that have been corrected here. 


\section{Continuous bodies, placements}

We use the definition of a continuous body-systems, placements, and frames of reference as presented in detail in Sections 4 and 5 of [BC]. Here, it is sufficient to use only one designated frame-space. a three-dimensional Euclidean space denoted by $\mathcal{E}$. Its translation space is denoted by $\mathcal{V}$. Also we only consider the case when the body-system consist only of one connected set, called simply "the body".

To give a precise definition of placements, one first has to introduce two sets:

(i) A set Fr consisting of connected fit regions in $\mathcal{E}$ that are candidates for regions occupied by the $\mathcal{B}$ when placed in $\mathcal{E}$. It serves well to take Fr to be the set of all fit regions in $\mathcal{E}$., as defined precisely in $[\mathrm{NV}]$, i We also consider the set $\overline{\mathrm{F}}$ of all closures of such fit regions. The set Fr not only includes all regions that an engineer can think of, but also some that only a mathematician can describe.

(ii) a set Tp of mappings which are candidates for changes of placements of the body in $\mathcal{E}$.

We call the members of Tp transplacements. Tp is characterized by the following conditions:

$\left(\mathbf{T}_{1}\right)$ Every $\lambda \in \mathrm{Tp}$ is an invertible mapping whose domain and codomain belong to Fr.

$\left(\mathbf{T}_{2}\right)$ For every $\lambda \in \mathrm{Tp}$, there is a $C^{2}$ - diffeomorphism $11 \phi: \mathcal{E} \longrightarrow \mathcal{E}$ such that $\lambda=\left.\phi\right|_{\operatorname{Dom} \lambda} ^{\text {Rng } \lambda}$.

In view of $\left(\mathrm{T}_{2}\right)$, each transplacement $\lambda$ has a unique extension $\bar{\lambda}$ whose domain and codomain belong to $\overline{F r}$.

The set $\mathrm{Pl}$ of all placements of $\mathcal{B}$ in $\mathcal{E}$ is characterized by the following conditions:

(B) Every $\kappa \in \mathrm{Pl}$ is an invertible mapping with Dom $\kappa=\mathcal{B}$ and $\mathrm{Rng} \kappa \in \mathrm{Fr}$.

$\left(\mathbf{B}_{2}\right)$ For all $\kappa, \gamma \in \mathrm{Pl}$ we have $\kappa \circ \gamma^{\leftarrow} \in \mathrm{Tp}$.

(B) For every $\kappa \in \mathrm{Pl}$ and $\lambda \in \operatorname{Tp}$ such that $\operatorname{Rng} \kappa=\operatorname{Dom} \lambda$ we have $\lambda \circ \kappa \in \mathrm{Pl}$.

\footnotetext{
${ }^{1}$ In most contexts, $C^{2}$ is good enough, but there may be situations where $C^{2}$ should be replaced by $C^{n}$ with $n>2$.
} 
We denote by $\Omega$ the set of all parts of $\mathcal{B}$ as defined in Sect. 4 of [BC]. Thus, for every $\mathcal{P} \in \Omega$, and every placement $\mu$, the fit region occupied by $\mathcal{P}$ is

$$
\mathcal{P}_{\mu}:=\mu_{>}(\mathcal{P}) \text {. }
$$

(See (5.7) in $[\mathrm{BC}]$.) The reduced boundary $\operatorname{Rby}\left(\mathcal{P}_{\kappa}\right)$ consists of those points $x \in$ Bdy at which an exterior unit normal $\mathbf{n}_{x} \in \mathrm{Sph} \mathcal{V}$ can be defined. (See Section 6 of $[\mathrm{NV}]$.)

\section{$3 \quad$ Load systems}

For the rest of this paper, we assume that a continuous body $\mathcal{B}$ and a frame-space $\mathcal{E}$ with translation space $\mathcal{V}$, as described in the previous section, are given. Again, we denote by $\Omega$ the set of all parts of $\mathcal{B}$.

Definition 3.1. A load $\mathbf{L}: \Omega \longrightarrow \mathcal{V}$ is an additive mapping, as defined in Sect.3 of [BC], with the following property: for every part $\mathcal{P} \in \Omega$ and every $\kappa \in \mathrm{Pl}$, there are continuous mappings $\mathbf{b}_{\kappa}:$ Clo $\mathcal{P}_{\kappa} \longrightarrow \mathcal{V}$ and $\mathbf{t}_{\kappa}:$ Rby $\mathcal{P}_{\kappa} \longrightarrow \mathcal{V}$ such that

$$
\mathbf{L}(\mathcal{P})=\int_{\mathcal{P}_{\kappa}} \mathbf{b}_{\kappa} \mathrm{d}_{\mathrm{vol}}+\int_{\mathrm{Clo} \mathcal{P}_{\kappa} \cap \mathrm{Rby} \mathcal{P}_{\kappa} \mathbf{t}_{\kappa} \mathrm{d}_{\text {area }} \quad \text { for all } \mathcal{P} \in \Omega}
$$

The set of all loads will be denoted by Ld.

In this definition, it is not difficult to show that one can replace "every $\kappa \in \operatorname{Pl} \mathcal{B}$ " by "some $\kappa \in \mathrm{Pl}$ " without changing the meaning. The condition $\left(\mathrm{T}_{2}\right)$ in Section 3 is crucial for proving this fact.

The first integral on the right side of (3.1) represents a body-load and the second a surface load. By (3.1), these loads have continuous densities. A more general definition should include

concentrated loads. We do not include them here because they would make the analysis too complicated.

The value of a load $\mathbf{L}$ on the entire body, i.e. $\mathbf{L}(\mathcal{B})$ is called the resultant of $\mathbf{L}$.

the set of all loads with resultants zero is denoted by

$$
\operatorname{Ld}_{0}:=\{\mathbf{L} \in \operatorname{Ld} \mid \mathbf{L}(\mathcal{B})=0\}
$$




\section{Elastic Responses}

We now assume that $\mathcal{B}$ is an elastic body, which means that it is a continuous body with additional structure as defined in [FFE]. The constitutive equation can be expressed with intrinsic stresses, Cauchy stresses, or Piola - Kirchhoff stresses. Here, it is useful to use a reference placement $\kappa$ and, for each $X \in \mathcal{B}$, the Piola - Kirchhoff stress

$$
\hat{T}_{R X}: \operatorname{Lis} \mathcal{V} \longrightarrow \operatorname{Lin} \mathcal{V}
$$

We say that the placement $\kappa$ is homogeneous if $\hat{T}_{R X}$ does not depend on $X$, so we simply denote it by $\hat{T}_{R}$. in this case. We say that $\kappa$ is a natural placement if it is homogeneous and if $\hat{T}_{R}\left(\mathbf{1}_{\mathcal{V}}\right)=\mathbf{0}$.

From now on, we assume that the reference placement $\kappa$ is homogenous . The stress function (4.1) is determined by the constitutive laws of elasticity.

We now consider the case when the material is hyper-elastic with a family $\left(\sigma_{\mu} \in \mathrm{C}^{1}(\operatorname{Lis}(\mathcal{V}), \mathbb{R}) \mid \mu \in\right.$ $\mathrm{Pl})$ of elastic energy functions. We also need the family of $\left(\rho_{\mu} \in \mathrm{C}^{1}\left(\operatorname{Lis}\left(\mathcal{V}, \mathbb{P}^{\times}\right) \mid \mu \in \mathrm{Pl}\right)\right.$ of mass-density functions, which assigns to each placement $\mu$ the volume per unit mass at each point in $\mathcal{B}_{\mu}$. We use the reference mass as defined in Sect.7 of $[\mathrm{BC}]$, so that $\rho_{\kappa}=1$.

We note that, for each $\mu$, the gradient of $\sigma_{\mu}$ is a mapping of the form $\nabla \sigma_{\mu}: \operatorname{Lis} \mathcal{V} \longrightarrow \operatorname{Lin}(\operatorname{Lin} \mathcal{V})$ because the formal codomain $\left.\operatorname{Lin}(\operatorname{Lin} \mathcal{V}, \mathbb{R})=\operatorname{Lin}(\operatorname{Lin} \mathcal{V})^{*}\right)$ can be identified with $\operatorname{Lin}(\operatorname{Lin} \mathcal{V})$, because $(\operatorname{Lin} \mathcal{V})^{*}$ can be identified with $(\operatorname{Lin} \mathcal{V})$ since $\mathcal{V}$ is an inner-product space.

The Piola-Kirchhoff Stress function $\hat{T}_{R}$ is then related to the elastic energy functions $\sigma_{\mu}$ by

$$
\hat{T}_{R}(\nabla(\chi))=\rho(\mu)\left(\nabla \sigma_{\mu}(\nabla \chi)\right)
$$

where

$$
\chi:=\mu \circ \kappa^{\leftarrow} .
$$

(In a more general theory of of thermomechanics. this relation is a consequence of the second law of thermodynamics. Originally. a version was proved in $[\mathrm{CN}]$, equation (5.4). Other versions are $(82.11)$ in $[\mathrm{NLFT}]$ and $(4.3)$ in $[\mathrm{FFE}]$.

In order to keep $\mathcal{B}$ in a given placement $\mu$, one must apply to it a certain load, the value at $\mu$ of an elastic load operator $\mathrm{El}: \mathrm{Pl} \longrightarrow \mathrm{Ld}$. This operator is determined by the stress operator $\tilde{T}_{\kappa}: \mathrm{Pl} \longrightarrow \mathrm{C}^{1}\left(\mathrm{Clo}_{\kappa}\right.$, Lin $\left.\mathcal{V}\right)$, defined by

$$
\tilde{T}_{\kappa}(\mu):=\hat{T}_{R}(\nabla(\chi))
$$


We then define the elastic body-force operator $\tilde{\mathbf{b}}_{\kappa}: \mathrm{Pl} \longrightarrow \mathrm{C}^{1}\left(\mathrm{Clo} \mathcal{B}_{\kappa} \mathcal{V}\right)$ by

$$
\tilde{\mathbf{b}}_{\kappa}(\mu):=-\operatorname{div}\left(\tilde{T}_{\kappa}(\mu)\right) \quad \text { for all } \quad \mu \in \mathrm{Pl},
$$

and the elastic surface-traction operator $\tilde{\mathbf{t}}_{\kappa}: \mathrm{Pl} \longrightarrow \mathrm{C}^{0}\left(\mathrm{Rby}_{\mathcal{B}_{\kappa}}, \mathcal{V}\right)$ by

$$
\tilde{\mathbf{t}}_{\kappa}(\mu):=\left.\tilde{T}_{\kappa}(\mu)\right|_{\operatorname{Rby}\left(\mathcal{B}_{\kappa}\right)} \mathbf{n}_{\kappa} \quad \text { for all } \quad \mu \in \mathrm{Pl},
$$

where $\mathbf{n}_{\kappa}: \operatorname{Rby} \mathcal{B} \longrightarrow \operatorname{Usph}(\mathcal{V})$ is the exterior unit normal function whose values are defined at the end Section 2.

The elastic load operator $\mathrm{El}: \mathrm{Pl} \longrightarrow \mathrm{Ld}$ is then defined by

$$
\operatorname{El}(\mu)(\mathcal{P})=\int_{\mathcal{P}_{\kappa}} \tilde{\mathbf{b}}_{\kappa}(\mu) \mathrm{d}_{v o l}+\int_{\mathrm{Clo} \mathcal{P}_{\kappa} \cap \mathrm{Rby} \mathcal{P}_{\kappa}} \tilde{\mathbf{t}}_{\kappa}(\mu) \mathrm{d}_{\text {area }} \quad \text { for all } \quad \mu \in \mathrm{Pl}, \quad \mathcal{P} \in \Omega .
$$

Of course, the right side of (4.7), contrary to appearance, does not depend on the choice of the reference placement $\kappa$. Also, we have $\operatorname{El}(\mu)(\mathcal{B})=0$, i.e., the resultant of $\operatorname{El}(\mu)$ is zero. Therefore, the codomain of $\mathrm{El}$ can be replaced by $\mathrm{Ld}_{0}$.

Elastic properties are frame- indifferent. Here, however, we deal with the interplay between an elastic body and its environment, which fails to be frame-indifferent because cannot be described without using an external frame, represented here by $\mathcal{E}$.

\section{Zero-load problems}

Let $\mathcal{B}$ be an elastic body with load operator El,, as described in the previous section. Given any load $\mathbf{L}_{0} \in \mathrm{Ld}_{0}$, one can consider the problem

$$
? \mu \in \mathrm{PL}, \quad \operatorname{El}(\mu)=\mathbf{L}_{0} \text {. }
$$

Such a problem is called dead-loading problem. If $\operatorname{El}(\mu) \neq \mathbf{L}_{0}$, such problems are physically unrealistic because it is difficult, in the real world, to create an environment that exerts a load-system on the body that does not vary with the placement.

If $\mathbf{L}_{0}=\mathbf{0}$, then (5.1) becomes the zero-load problem

$$
? \mu \in \mathrm{Pl}, \operatorname{El}(\mu)=\mathbf{0} \text {. }
$$


which has the following vivid physical interpretation: Take the body, in some given reference placement $\kappa$, and then "let it go" so as to end up completely free of external forces. What placements $\mu$, if any, can it assume?

If $\mu \in \mathrm{Pl}$ is a solution of (5.2), and $\gamma$ a congruence from $\mathcal{B}_{\mu}$ onto a subset of $\mathcal{E}$, then $\alpha \circ \mu$ is again a solution of $(5.2)$. We say that two solutions of $(5,2)$ that are related by such are congruence equivalent solutions.

We assume now that $\mathcal{B}$ has natural placements $\kappa \in \mathrm{Pl}$. It is evident that these are solutions to the zero-load problem. Physical experience tells us, however, that they need not be the only such solutions. For example, if $\kappa$ is a natural placement and the region $\mathcal{B}_{\kappa}$ occupied by a body made of rubber has the shape of a hemispherical shell, one can expect a solution $\mu$ to (5.2), not equivalent to $\kappa$, that corresponds to an "eversion" of the shell (see Fig. 1).

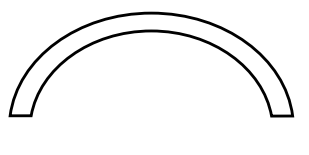

$\kappa$

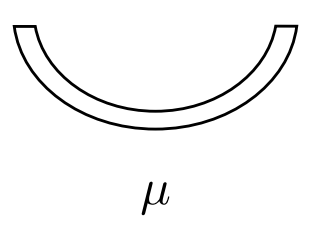

Figure 1: eversion

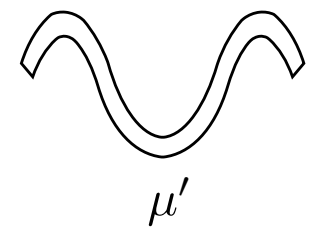

$\mu$

If the shell is thin enough, one can experimentally produce a third solution $\mu^{\prime}$, corresponding to eversion of only the middle of the shell, as shown in Figure 1. Actually, if the eversion or partial eversion is produced by pushing down the middle while holding the rim along the circle, one can produce additional solutions to (5.2). They correspond to the onset of "popping" into the everted or partially everted placements and are physically unstable.

A more complicated situation can be expected when the region $\mathcal{B}_{\kappa}$ occupied by $\mathcal{B}$ in the natural placement $\kappa$ has the shape of a "rubber mat" with many hemispherical indentations (see Fig. 2).

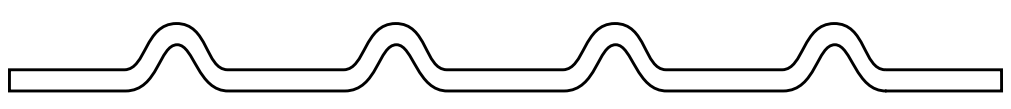

Figure 2: rubber mat

One expects that there are many inequivalent solutions to (5.2), each corresponding to "eversion" of a prescribed subset of the set of indentations. Thus, if there are $n$ indentations, one expects 
at least $2^{n}$ solutions. In addition, there are physically unstable solutions.

Remark In the examples above, the region $\mathcal{B}_{\kappa}$ occupied by the body in its natural placements fails to be convex. I offer the following conjecture: If the stress function of an elastic body satisfies conditions appropriate for materials such as rubber and if the body occupies a convex region in its natural placements, then these natural placements are the only solutions to the zero-load problem.

If an elastic body has no natural placement, there are circumstances when one can expect that the zero-load problem has no solutions at all. Consider, for example, two bodies $\mathcal{B}_{1}$ and $\mathcal{B}_{2}$ with natural placements $\kappa_{1}$ and $\kappa_{2}$ as shown in Figure 3.

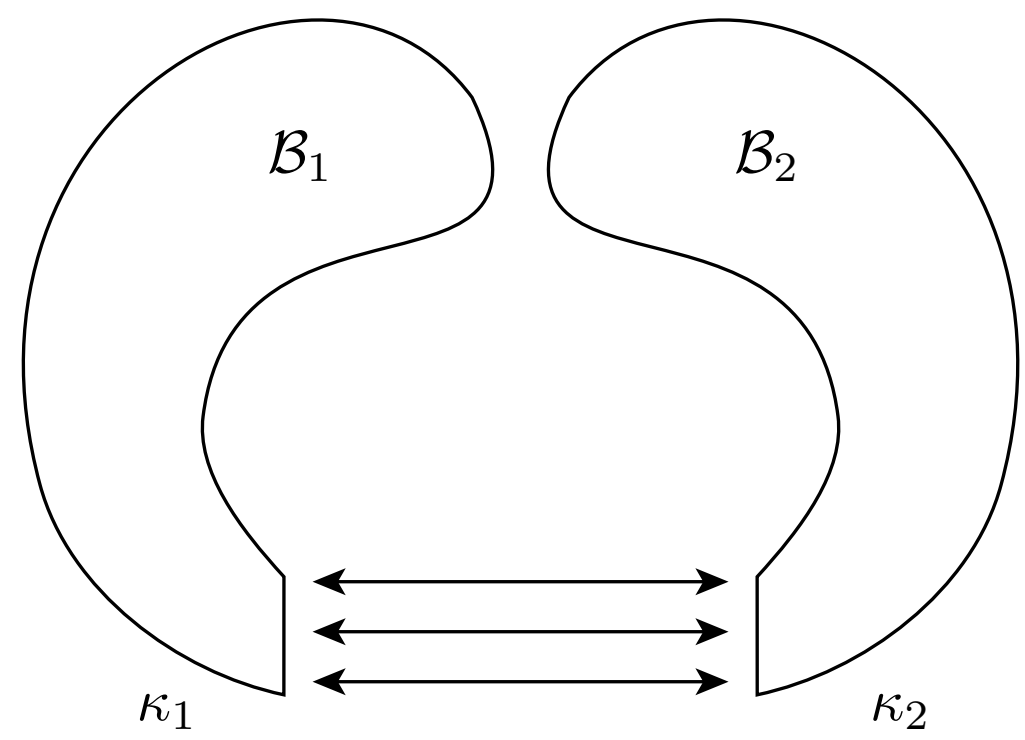

Figure 3:

Assume that the two bodies consist of the same material, and hence that $\hat{T}_{R_{1}}=\hat{T}_{R_{2}}$. One can then "glue" $\mathcal{B}_{1}$ and $\mathcal{B}_{2}$ together as indicated in Figure 3 to obtain a single body $\mathcal{B}$ which has no homogeneous placement However, $\mathcal{B}$ has no natural placements because, before gluing $\mathcal{B}_{1}$ and $\mathcal{B}_{2}$ together, one must bend their upper parts out of the way, so that every placement $\mu$ of $\mathcal{B}$ fails to be homogeneous. Hence the problem (5.2) of has no solutions at all in this case, 


\section{Environmental reactions}

In general, if the elastic body $\mathcal{B}$ is placed the environment represented by the Euclidean space $\mathcal{E}$, the environment will exert forces on $\mathcal{B}$, and these forces will depend on the placement of $\mathcal{B}$. These forces are specified by the prescription of an environmental-reaction operator Er : PL $\longrightarrow \mathrm{Ld}$, whose value $\operatorname{Er}(\mu)$ gives the load that the environment exerts on $\mathcal{B}$ when placed in $\mu$.

Given a load $\mathbf{L}_{0}$ with zero resultant, the problem

$$
? \mu \in \mathrm{Pl}, \quad \operatorname{Er}(\mu)=\operatorname{El}(\mu)
$$

then has the following physical interpretation: Put the elastic body $\mathcal{B}$, with the elastic load operator El, in an environment described by the environmental load operator Er and then "let it go". What placements $\mu$, if any, can it assume.

Given any homogeneous reference placement $\kappa$, one can describe Er by an environmental body-force operator $\overline{\mathbf{b}}_{\kappa}: \mathrm{Pl} \longrightarrow \mathrm{C}^{0}\left(\mathrm{Clo}_{\kappa}, \mathcal{V}\right)$ and an environmental surface-traction operator $\overline{\mathbf{t}}_{\kappa}: \mathrm{Pl} \longrightarrow \mathrm{C}^{0}\left(\mathrm{Rby} \mathcal{B}_{\kappa}, \mathcal{V}\right)$ such that

$$
\operatorname{Er}(\mu)(\mathcal{P})=\int_{\mathcal{P}_{\kappa}} \tilde{\mathbf{b}}_{\kappa}(\mu) \mathrm{d}_{\text {vol }}+\int_{\mathrm{Clo}_{\kappa} \cap \mathcal{P}_{\kappa} \cap \mathcal{P}_{\kappa}} \overline{\mathbf{t}}_{\kappa}(\mu) \mathrm{d}_{\text {area }} \quad \text { for all } \mathcal{P} \in \Omega
$$

In view of (4.6), the problem (6.1) is then equivalent to

$$
? \quad \mu \in \mathrm{Pl}, \quad \overline{\mathbf{b}}_{\kappa}(\mu)=\tilde{\mathbf{b}}_{\kappa}(\mu), \quad \overline{\mathbf{t}}_{\kappa}(\mu)=\tilde{\mathbf{t}}_{\kappa}(\mu)
$$

In most physical situations, the environmental body-force operator is local in the sense that it is determined by by a family $\left(\mathbf{b}_{\mu} \in \mathrm{C}^{0}\left(\mathrm{Clo}_{\mu}, \mathcal{V}\right) \mid \mu \in \mathrm{Pl}\right)$ such that

$$
\int_{\mathcal{P}_{\kappa}} \overline{\mathbf{b}}_{\kappa} \mathrm{d}_{v o l}=\int_{\mathcal{P}_{\mu}} \mathbf{b}_{\mu} \mathrm{d}_{v o l} \quad \text { for all } \quad \mu \in \mathrm{Pl}
$$

For each $\mu \in \mathrm{Pl}, \quad \mathbf{b}_{\mu} \in \mathrm{C}^{0}\left(\mathrm{Clo}_{\mu}, \mathcal{V}\right)$ gives the environmental body force per unit volume when $\mathcal{B}$ is placed in the space $\mathcal{E}$ by the placement $\mu$. Using the Theorem on Transformation of Volume Integrals, it follows from $(6,3)$ that

$$
\overline{\mathbf{b}}_{\kappa}=|\operatorname{det}(\nabla \chi)|\left(\mathbf{b}_{\mu} \circ \chi\right),
$$

where the transplacement $\chi$ is defined by (4.3). 
Using (4.3) and (6.5), We see that the condition $\overline{\mathbf{b}}_{\kappa}(\mu)=\tilde{\mathbf{b}}_{\kappa}(\mu)$ of the problem (6.3) becomes

$$
\operatorname{div}\left(\tilde{T}_{\kappa}(\mu)\right)+\overline{\mathbf{b}}_{\kappa}=0 .
$$

Using (4.2) and (6.6), we obtain the classical differential equation of finite elasticity

$$
\operatorname{div}\left(\hat{T}_{R}(\nabla \chi)\right)+\overline{\mathbf{b}}_{\kappa}=0
$$

where $\overline{\mathbf{b}}_{\kappa}$ is given by (6.5). This is a differential equation for the trasplacement $\chi$.

If the only environmental body force is gravitational or electrostatic with a potential $\phi \in$ $\mathrm{C}^{1}(\mathcal{E}, \mathcal{V})$, and if the gravitational mass density or electric charge density $\rho_{\mu}:$ Clo $\mathcal{B}_{\mu} \longrightarrow \mathbb{R}$ is given, then $\mathbf{b}_{\mu}$ has the form

$$
\mathbf{b}_{\mu}(x):=-\rho_{\mu}(x)(\nabla \phi)(x) \quad \text { for all } x \in \mathrm{Clo}_{\mu}
$$

In some physical situations, the environmental surface-traction operator is local in the sense that it is determined by by a family $\left(\mathbf{t}_{\mu} \in \mathrm{C}^{0}\left(\mathrm{Rby} \mathcal{B}_{\mu}, \mathcal{V}\right) \mid \mu \in \mathrm{Pl}\right)$ such that

$$
\int_{\text {Rby }_{\kappa}} \overline{\mathbf{t}}_{\kappa} \mathrm{d}_{\text {area }}=\int_{\mathrm{Rby}_{\boldsymbol{P}}} \mathbf{t}_{\mu} \mathrm{d}_{\text {area }} \quad \text { for all } \quad \mu \in \mathrm{Pl} .
$$

For each $\mu \in \mathrm{Pl}, \quad \mathbf{t}_{\mu} \in \mathrm{C}^{0}\left(\mathrm{Rby}_{\mu}, \mathcal{V}\right)$ gives the environmental surface traction per unit area when $\mathcal{B}$ is placed in the space $\mathcal{E}$ by the placement $\mu$. Using the Theorem on Transformation of Surface Integrals, it follows from (6.9) that

$$
\overline{\mathbf{t}}_{\kappa}=|\operatorname{det}(\nabla \chi)|\left(\mathbf{t}_{\mu} \circ \chi\right)(\nabla \chi)^{-\top} .
$$

If this is the case, the condition $\overline{\mathbf{t}}_{\kappa}(\mu)=\tilde{\mathbf{t}}_{\kappa}(\mu)$ of (6.3) becomes a boundary condition, which, by (4.2) and (4.4), has the explicit form

$$
\hat{T}_{R}\left(\left.\nabla(\chi)\right|_{\mathrm{Rby} \mathcal{B}_{\kappa}}\right) \mathbf{n}_{\kappa}=\overline{\mathbf{t}}_{\kappa}
$$

where $\overline{\mathbf{t}}_{\kappa}$ is given by $(6.10)$.

In many, perhaps most, physical situations, the environmental surface-traction operator is not local and hence does not give rise to a boundary condition in the conventional sense. An example is the cavity problem. In this case, we assume that Bdy $\mathcal{B}_{\kappa}$ has two connected components $\mathcal{S}_{c_{\kappa}}$ and $\mathcal{S}_{e_{\kappa}}$. The first is the surface of a connected cavity surrounded by $\mathcal{B}_{\kappa}$ and the second the exterior 
boundary of $\mathcal{B}_{\kappa}$. In view of the conditions $\left(\mathrm{T}_{2}\right)$ and $\left(\mathrm{B}_{2}\right)$ in Section 2 , the assumption above on $\mathcal{B}_{\kappa}$ remains valid if the reference placement $\kappa$ is replaced by an arbitrary placement $\mu \in \mathrm{Pl}$.

Let $\mu \in \mathrm{Pl}$ be given. Then $\mathcal{S}_{c_{\mu}}$ is the boundary of a exactly one connected open region whose volume we denote by $v(\mu)$. Thus we have defined a mapping $v: \mathrm{Pl} \longrightarrow \mathbb{P}^{\times}$.

We now assume that the cavity $\mathcal{S}_{c_{\mu}}$ is filled by a compressible homogeneous gas. Assuming that the temperature is constant, the pressure in the cavity depends only on its volume and this dependence is described by a function $\pi: \mathbb{P}^{\times} \longrightarrow \mathbb{P}^{\times}$, usually antitone. Also, we assume that the outside pressure on the exterior boundary $\mathcal{S}_{e_{\mu}}$ has a fixed value $p \in \mathbb{P}^{\times}$, independent of the placement $\mu$. For example, $p$ may be the atmospheric pressure. Using (6.10), we find that the environmental surface traction is given by

$$
\left.\overline{\mathbf{t}}_{\kappa}\right|_{\mathcal{S}_{e_{\kappa}}}:=p|\operatorname{det}(\nabla \chi)|\left(\mathbf{t}_{\mu} \circ \chi\right)(\nabla \chi)^{-\top} .
$$

and

$$
\left.\overline{\mathbf{t}}_{\kappa}\right|_{\mathcal{S}_{c_{\kappa}}}:=-\pi\left(v(\mu)|\operatorname{det}(\nabla \chi)|\left(\mathbf{t}_{\mu} \circ \chi\right)(\nabla \chi)^{-\top},\right.
$$

where the transplacement $\chi$ is defined by (4.3).

Of particular practical interest is the case when $\mathcal{S}_{c_{\kappa}}$ and $\mathcal{S}_{e_{\kappa}}$ are concentric spheres, the first one having the smaller radius and $\mathcal{B}_{\kappa}$ being the region between them. In this case, $\mathcal{B}_{\kappa}$ looks like a basketball or a soccer-ball. We then call (6.1) the basketball problem.

Another case of practical interest is the one when both $\mathcal{S}_{c_{\kappa}}$ and $\mathcal{S}_{e_{\kappa}}$ are tori, i.,e.,surfaces of revolution generated by revolving a circle in $\mathcal{E}$ about an axis in the same planes as the revolving circle. We assume that this axis is the same for both tori and that the the first torus is included in the open region bounded by the second torus. In this case , $\mathcal{B}_{\kappa}$ looks like a tire. We then call (6.1) the tire problem.

\section{Stability}

There is a vast literature on elastic stability as described in Section 89 of [NLFT]. However, this literature deals only with local boundary conditions. It is not difficult to generalize elastic stability to the case when local boundary conditions are replaced by environmental operators as described 
in the previous section This is shown in Section 8 of [Rio]. We now assume that $\mathcal{B}$ is hyper-elastic. Then the total elastic energy when $\mathcal{B}$ is in a given placement $\mu$ is given by

$$
\Sigma(\mu)=\int_{\mathcal{B}_{\mu}} \sigma(\mu)
$$

We also assume that the environmental-reaction operator discussed in Section 6 is conservative in the sense that it can be derived from a potential energy $\Pi: \mathrm{Pl} \longrightarrow \mathbb{R}$. This is the case, for example, when the environmental body force is purely gravitational or electrical, as described in the previous section.

In this case, a solution $\mu$ of the problem (6.1) is locally stable if there is a neighborhood $\Lambda$ of $\mu \in \mathrm{Pl}$ such that

$$
\Sigma(\mu)-\Pi(\mu) \leq \Sigma(\lambda)-\Pi(\lambda) \quad \text { for all } \quad \lambda \in \Lambda,
$$

i.e. that $\Sigma-\Pi$ has a local minimum at $\mu$.

\section{Outlook}

Here are some issues that may be considered in future research:

1) Internal constraints, incompressibility. This subject is treated in detail in Section 30 of [NLFT] . The simplest and most important of these is incompressibility. It should not be difficult to modify the content of this paper when the elastic body $\mathcal{B}$ is incompressible.

2) External constraints. The most important of these is a Confinement, which is determined by prescribing an open connected subset $\mathcal{C}$ of $\mathcal{E}$ with a piecewise smooth boundary. We may think of this boundary as consisting of a rigid flat or curved sheet. If $\mathcal{C}$ is a half-space, then its boundary corresponds to a rigid plane sheet. The set $\mathrm{Pl}$ should then be reduced to

$$
\left.\mathrm{Pl}_{\mathrm{C}}:=\left\{\mu \in \mathrm{Pl} \mid \mathcal{B}_{\mu} \subset \mathcal{C}\right)\right\}
$$

the set of admissible placements.

If $\mu \in \mathrm{Pl}_{\mathrm{c}}$ touches the sheet represented by $\mathrm{BdyC}$, it may stick to it, glide freely, or glide with friction. In the first case we get what is usually called a boundary condition of place. The other two cases should be investigated. Some of that was done in Section 7 of [Rio]. 
There is also the possibility that there are no stable solutions in which no $\mu \in \mathrm{Pl}_{\mathrm{c}}$ touches the sheet represented by $\mathrm{BdyC}$. (See, for example $[\mathrm{FT}]$ and $\mathrm{MH}]$.)

3) Solutions. This paper only describes schemes for posing problems, but not for actually solving them. I believe that one can obtain more or less explicit solutions in some cases, for example for the basketball problem and the tire problem, especially when the body in the reference placement is isotropic.

4) Quasi-static motions. One may investigate motions of an elastic body that are so slow that inertial effects can be neglected. These are time families $\left(\mu_{t} \in \mathrm{Pl} \mid \mathrm{t} \in[0, \mathrm{~d}]\right)$ of duration $d$. The elastic Load operator $\mathrm{El}_{t}$ varies with $t \in[0, d]$ but the environmental-reaction operator Er remains constant.

The beginning of such an investigation is described in Section 8 of [Rio] .

5) Zero-load problems. Strictly speaking, one cannot make experiments on the surface the earth that correspond to solutions to zero-load problems, because there is always the earth's gravitational force. One would have to make such experiments in the weightless environment of a space station. However, if the material of the elastic body has small weight, the zero-load problems may be a good approximation.

It may be useful to consider the case when the environmental reaction is reduced to a constant weight per unit volume.

5) Thermal effects. Here, as in most treatments of elastostatics, thermal effects are neglected, So the theory applies only to the case when changes are so slow that the temperature changes very little. It would be interesting to find a generalization in which heat conduction is taken into account.

6) Surface tension. In this paper, we assume that there is no surface tension or, at least, that it is negligible. It would be interesting to develop a conceptual scheme that includes the effect of surface tension.

Acknowledgement I am grateful to Roger Fosdick and Eliot Fried for valuable suggestions and proof reading. 


\section{References}

[CIM] Noll, W. : The Conceptual Infrastructure of Mathematics, published as A 2) 0n Noll's website math.cmu.edu/ wnog/noll.

[FDSI] Noll, W. : Finite-Dimensional Spaces: Algebra, Geometry, and Analysis, Vol.I, 393 pages, published on Noll's website math.cmu.edu/ wn0g/noll . ( Originally published by Martinus Nijhoff Publishers in 1987.)

[BC] Noll, W \& Seguin, B.: Basic Concepts of Thermomechanics, Journal of Elasticity 101,121151(2009).

[NLFT] Truesdell, C. \& Noll, W.: The Non-Linear Field Theories of Mechanics, third Edition, 602 pages. Springer-Verlag, 2004. (The first edition appeared in 1965.)

[FFE] Noll, W: A Frame-Free Formulation of Elasticity, Journal of Elasticity 83 , 291-307 (2006).

[FC] Noll, W. : Five Contributions to Natural Pilosophy, 73 pages 2004, published on the website math.cmu.edu/ wnOg/noll .

[Rio] Noll, W: A general framework for problems in the statics of finite elasticity, Contemporary Development in Continuum Mechanics and Partial Differential Equations, pp. 363-387, NorthHolland Mathematics Studies 30, (1978).

[NV] Noll, W. \& Virga, E.: Fit Regions and Functions of Bounded Variation, Archive for Rational Mechanics and Analysis 102, 1-21 (1988).

[CN] Coleman, B.D. \& Noll, W.: The thermodynamics of elastic materials with heat conduction and viscosity, Archive for Rational Mechanics and Analysis, 13, 167-178 (1963).

[FT] Fried, E. \& Todres, R. E.: Mind the Gap: The Shape of the Free Surface of a Rubber-Like Material in Proximity to a Rigid Contactor, Journal of Elasticity 80 , 97-151 (2005).

[MH] . Mönch, W. \& Herminghaus, S.: Elastic instability of rubber films between solid bodies. Europhys. Lett., 53 (4), pp. 525-531 (2001). 